\begin{tabular}{|c|c|c|c|l|l|}
\hline \multirow{2}{*}{$\sim$} & H E L & $\mathbf{1 2 3 0 4}$ & \multirow{2}{*}{ WILEY } & Dispatch: 25.2.16 & CE: Keerthana \\
\cline { 2 - 3 } \cline { 5 - 5 } & Journal Code & Manuscript No. & & No. of pages: 7 & PE: Pravin Kumar A \\
\hline
\end{tabular}

Helicobacter ISSN 1523-5378

doi: $10.1111 /$ hel. 12304

\title{
Usefulness of Housekeeping Genes for the Diagnosis of Helicobacter pylori Infection, Strain Discrimination and Detection of Multiple Infection
}

Montserrat Palau,* Marcos Kulmann,* María José Ramírez-Lázaro, ${ }^{\dagger+\$}$ Sergio Lario, ${ }^{\dagger+\$}$ María Elisa Quilez, ${ }^{\dagger+\star}$

Rafael Campo, ${ }^{\dagger, \star}$ Núria Piqué,* Xavier Calvet ${ }^{\dagger, \star}$ and David Miñana-Galbis*

*Departament de Microbiologia i Parasitologia Sanitàries, Facultat de Farmàcia, Universitat de Barcelona, Barcelona, Catalonia, Spain, ${ }^{\dagger}$ Digestive Diseases Service, Hospital de Sabadell, Institut Universitari Parc Taulí, Departament de Medicina, Universitat Autònoma de Barcelona, Sabadell, 3 Madrid, Spain

\section{Keywords}

Helicobacter pylori, housekeeping genes, detection, genotyping, multiple infection.

Reprint requests to: Prof. Dr. David MiñanaGalbis, Departament de Microbiologia i Parasitologia Sanitàries, Facultat de Farmàcia, Universitat de Barcelona, Av. Joan XXIII s/n, 08028 Barcelona, Catalonia, Spain. E-mail: davidminyana@ub.edu

\begin{abstract}
Background: Helicobacter pylori infects human stomachs of over half the world's population, evades the immune response and establishes a chronic infection. Although most people remains asymptomatic, duodenal and gastric ulcers, MALT lymphoma and progression to gastric cancer could be developed. Several virulence factors such as flagella, lipopolysaccharide, adhesins and especially the vacuolating cytotoxin VacA and the oncoprotein CagA have been described for $H$. pylori. Despite the extensive published data on H. pylori, more research is needed to determine new virulence markers, the exact mode of transmission or the role of multiple infection.

Materials and Methods: Amplification and sequencing of six housekeeping genes (amiA, cgt, cpn60, cpn70, dnaJ, and luxS) related to H. pylori pathogenesis have been performed in order to evaluate their usefulness for the specific detection of $H$. pylori, the genetic discrimination at strain level and the detection of multiple infection. A total of $52 \mathrm{H}$. pylori clones, isolated from 14 gastric biopsies from 11 patients, were analyzed for this purpose.

Results: All genes were specifically amplified for H. pylori and all clones isolated from different patients were discriminated, with gene distances ranged from 0.9 to $7.8 \%$. Although most clones isolated from the same patient showed identical gene sequences, an event of multiple infection was detected in all the genes and microevolution events were showed for amiA and cpn60 genes.

Conclusions: These results suggested that housekeeping genes could be useful for H. pylori detection and to elucidate the mode of transmission and the relevance of the multiple infection.
\end{abstract}

Helicobacter pylori chronically infects more than half of the world's population because the host immune response fails to eliminate the infection. In most infected people, the bacterium acts as a commensal organism inducing chronic asymptomatic gastritis that can last for life. In other cases, however, it is responsible for a heavy toll of morbidity and mortality as a consequence of peptic ulcers and gastric cancer. Chronic gastritis may progress to intestinal metaplasia, dysplasia and eventually gastric cancer. This multi-step process is known as the Correa pathway [1]. The clinical outcome of $H$. pylori infection depends on strain virulence, host response, and environmental factors.

Several virulence factors have been identified in H. pylori: gastric colonization mediated by flagella, urease, outer membrane proteins (OMPs), phospholipids, glycolipids, and other adhesins ( $b a b A, h p A$, napA, $s a b A$, etc.), toxins such as lipopolysaccharide (LPS) and the vacuolating cytotoxin VacA, a type IV secretion system encoded by the cag pathogenicity island (cagPAI) containing the effector protein CagA, a $\gamma$-Glutamyl transpeptidase (GGT) associated with colonization and 
cell apoptosis, the duodenal ulcer-promoting gene A $(\operatorname{dup} A)$, and others [2-4]. Among virulence factors identified in H.pylori, cagA, and vacA genes are the main virulence markers as shown in an study conducted by [5], in which patients infected with high virulence strains (cagA+, vacA slil-ml) had a higher risk of progression to preneoplastic lesions and gastric cancer in comparison with patients infected with low virulence strains.

Strains of H.pylori exhibit considerable genetic diversity following a panmictic (non-clonal) population structure due to horizontal gene transfer and frequent recombination. In contrast, since H. pylori does not spread epidemically, phylogenetic studies based on multilocus sequence analysis (MLSA) of housekeeping genes are contributing to trace human migrations $[6,7]$. On the other hand, intrapatient diversity of $H$. pylori needs to be reevaluated because results about the prevalence of multiple infection are controversial, and it could have implications related to the mode of transmission, antibiotic resistance and virulence of $H$. pylori $[2,8,9]$. Moreover, there is a lack of studies regarding H. pylori detection from specimens other than gastric biopsies [10].

Other genes have likewise been related to $H$. pylori pathogenesis: amiA (N-acetylmuramoyl-L-alanine amidase) and luxs (autoinducer-2 synthase) genes are involved in biofilm formation although the former is also related to bacterial adhesion, cell morphology and immune escape [11]. Others such the cholesterol- $\alpha$-glucosyltransferase gene (cgt or capJ) glycosilates host cholesterol contributing to pathogenicity and antimicrobial resistance while cpn40 (dnaJ), cpn60 (groEL), and cpn70 (dnaK) genes have been suggested as a risk factor for oncogenesis and are recommended as serological markers of $H$. pylori infection [3,12,13].

In this study, H. pylori specific PCR amplification and sequencing of amiA, cgt, cpn60, cpn70, dnaJ, and luxS genes have been developed in order to evaluate their usefulness in the detection of $H$. pylori infection, to genetic discrimination at strain level, to detection of multiple infection and to MLSA.

\section{Materials and Methods}

\section{Helicobacter pylori Clones and DNA Extraction}

Ten H. pylori strains (APP134, B247, B271, B319, B355, B491, B508S, B508T, CRL122, and SVC135) from the H. pylori collection of the Digestive Diseases Department of the Hospital Taulí (Sabadell, Barcelona, Spain) were included in this study. Outpatients sent to the Endoscopy Unit of the Hospital Taulí for evaluation of dys- peptic symptoms from February 2006 to November 2015 were recruited in this collection. Patients were contacted prior to the endoscopy and were asked to participate. Before the endoscopy, the patients signed informed consent. During endoscopy, antral and corpus biopsies were obtained for histology, rapid urease test (RUT) and molecular studies. Isolation, culture and identification of $H$. pylori were performed after a positive RUT test. The RUT biopsy was plated on Pylori Agar (bioMérieux) in microaerophilic conditions in 4 microaerophilic jars (Jar Gassing System; Don Whitley Scientific Limited, UK). After a maximum of a week, 5 H. pylori isolates were subcultured on Columbia plates (bioMérieux) and identified by colony morphology, Gram-negative staining and a positive result for urease, catalase, and oxidase tests. The strains were frozen in Brucella Broth with $10 \%$ glycerol and stored at $-80{ }^{\circ} \mathrm{C}$ until analysis [14].

The strains were recovered on Columbia agar with $5 \%$ sheep blood (bioMérieux) and incubated at $37{ }^{\circ} \mathrm{C}$ under microaerophilic conditions. These strains were previously isolated from antral biopsies of different patients, except for B508S and B508T, which were isolated from the same patient, B508S from normal tissue and B508T from gastric adenocarcinoma (Table 1). For this study, these H. pylori strains were not considered as pure cultures because they were originally obtained by picking up most of the growth on Pylori agar (bioMérieux) after seeding of gastric biopsies, and not

Table 1 Samples included in this study

\begin{tabular}{lll}
\hline $\begin{array}{l}\text { Gastric } \\
\text { biopsies } \\
(n=14)\end{array}$ & $\begin{array}{l}\text { Endoscopic/Histopathological } \\
\text { diagnosis }\end{array}$ & $\begin{array}{l}\text { Clones analyzed } \\
(\mathrm{n}=52)\end{array}$ \\
\hline APP134 & Duodenal ulcer/moderate gastritis & APP134-1, -2, -3 \\
B247 & Neoplasia/adenocarcinoma & B247-1, -2, -3 \\
B271 & Duodenal ulcer/moderate gastritis & B271-1, -2, -3 \\
B319 & Normal/mild gastritis & B319-1, -2, -3 \\
B355 & Duodenal ulcer/mild gastritis & B355-1, -2, -3 \\
B491 & Neoplasia/adenocarcinoma & B491-1, -2, -3 \\
B5085 & Neoplasia/adenocarcinoma & B508S-1, -2, -3, -4, \\
& & $-5,-6$ \\
B508T & Neoplasia/adenocarcinoma & B508T-2A $,-2 B,-3,-4$, \\
& & $-5,-6$ \\
B657A & Normal/mild gastritis & B657A-1, -2, -3, -4 \\
B657C & Normal/mild gastritis & B657C-1, -2, -3, -4 \\
B659A & Normal/moderate gastritis & B659A-1, -2, -3, -4 \\
B659C & Normal/moderate gastritis & B659C-1, -2, -3, -4 \\
CRL122 & Normal/mild gastritis & CRL122-1, -2, -3 \\
SVC135 & Normal/moderate gastritis & SVC135-1, -2, -3 \\
\hline
\end{tabular}

${ }^{a}$ Gastric biopsies obtained from normal tissue of patients with adenocarcinoma. 
from individual colonies. In order to obtain pure cultures, between three and six isolated colonies were selected from each blood agar culture.

Helicobacter pylori colonies were also isolated from antral and corpus biopsies of two different patients (B657A/B657C and B659A/B659C) (Table 1) seeded onto Pylori agar.

Helicobacter pylori strains ATCC 49503 and ATCC 51932 were also included as controls in the present study.

Subcultures of individual colonies were performed on Columbia blood agar or Brucella agar (BD Diagnostics) supplemented with $10 \%$ fetal bovine serum (FBS; 6 Invitrogen).

\section{DNA Extraction, PCR Amplification and Sequencing}

DNA of each clone was extracted from $4 \mathrm{ml}$ of cultures in Brucella broth supplemented with 10\% FBS following the manufacturers' instructions (REAL pure geno7 mic DNA extraction kit).

Primers for $H$. pylori specific PCR amplification and sequencing of the housekeeping genes amiA, cgt, cpn60, cpn70, dnaJ, and luxS (Table 2) were designed from 43 H. pylori complete genome sequences (see Table S1). To evaluate the specificity of the PCR assays, the following four strains, belonging to different bacterial species, were included as negative controls: Campylobacter jejuni ATCC 33291, Pseudomonas aeruginosa 42A2, Aeromonas molluscorum 848T $\mathrm{T}^{\mathrm{T}}$, and Aeribacillus pallidus DR03.

PCR amplifications were carried out in a total volume of $50 \mu \mathrm{L}$ containing $50 \mathrm{mM} \mathrm{KCl}, 15 \mathrm{mM}$ Tris/HCl, $\mathrm{pH}$ 8.0, $2.5 \mathrm{mM} \mathrm{MgCl}_{2}, 0.2 \mathrm{mM}$ dNTPs (Amersham Biosciences), $2.5 \mathrm{U}$ AmpliTaq Gold DNA polymerase
(Applied Biosystems), $40 \mathrm{pmol}$ of each primer (Sigma), and 250 ng DNA. The reaction mixtures were subjected to the following thermal cycling program in a 2720 Thermal Cycler (Applied Biosystems): denaturation at $95{ }^{\circ} \mathrm{C}$ for 5 minutes, 35 cycles of $95^{\circ} \mathrm{C}$ for 1 minutes, 54-64 ${ }^{\circ} \mathrm{C}$ (Table 2) for 1 minute and $72{ }^{\circ} \mathrm{C}$ for 1 minute and a final extension step at $72{ }^{\circ} \mathrm{C}$ for 5 minutes.

The amplified products were purified using the MSB $^{\circledR}$ Spin PCRapace kit (Stratec) or the ExoSAP-IT ${ }^{\circledR}$ (Affymetrix) and sequencing was performed using the ABI PRISM BigDye Terminator v3.1 Cycle Sequencing kit (Applied Biosystems) with the following thermal cycling conditions: $96{ }^{\circ} \mathrm{C}$ for 1 minute and 25 cycles of $96{ }^{\circ} \mathrm{C}$ for 10 seconds, $50{ }^{\circ} \mathrm{C}$ for 5 seconds and $60{ }^{\circ} \mathrm{C}$ for 4 minutes. Nucleotide sequences were determined in an ABI PRISM 3730 DNA analyzer by the Genomics Unit of Scientific and Technological Centers from University of Barcelona (CCiTUB).

\section{Data Analyses}

Partial sequences of amiA, cgt, cpn60, cpn70, dnaJ, and luxs genes were aligned independently and phylogenetic trees were constructed by neighbor-joining method and Jukes-Cantor distance estimation model using MEGA 6.0 [15]. Concatenated sequences of all genes obtained by the online sequence toolbox FaBox [16] were also analyzed (Table 3). The topological robustness of the phylogenetic trees was evaluated by a bootstrap analysis through 1000 replicates.

\section{Results}

A total of $52 \mathrm{H}$. pylori clones were isolated from 14 gastric biopsies from 11 patients (Table 1).

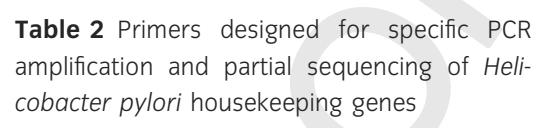

\begin{tabular}{|c|c|c|c|c|c|}
\hline Gene & Length $(p b)^{a}$ & Primer & Sequence $5^{\prime} \rightarrow 3^{\prime}$ & $\mathrm{Ta}$ & Amplicon (pb) \\
\hline amiA & 1323 & $\begin{array}{l}\text { amiA-658 } \\
\text { amiA-1292 }\end{array}$ & $\begin{array}{l}\text { GTTTTRGACGCYGGGCATGG } \\
\text { CCATCAGCAATGCCCTTAGC }\end{array}$ & $64^{\circ} \mathrm{C}$ & 635 \\
\hline $\operatorname{cgt}$ & 1170 & $\begin{array}{l}\text { cgt-252 } \\
\text { cgt-866 }\end{array}$ & $\begin{array}{l}\text { GGCTTTTAAAGGGAGCGGATA } \\
\text { ATCGCTTCGCTYTCCACATT }\end{array}$ & $60^{\circ} \mathrm{C}$ & 615 \\
\hline cpn60 & 1641 & $\begin{array}{l}\text { Hp156 } \\
\text { Hp956 }\end{array}$ & $\begin{array}{l}\text { CGTGAGCGTGGCTAARGAG } \\
\text { GCTTTGCCTAAAAACTCYACTT }\end{array}$ & $54^{\circ} \mathrm{C}$ & 801 \\
\hline cpn70 & 1863 & $\begin{array}{l}\text { cpn70-982 } \\
\text { cpn70-1593 }\end{array}$ & $\begin{array}{l}\text { ATTTCAGAAGTGGTGATGGT } \\
\text { GTTTCTCGCTTCAATCACTT }\end{array}$ & $54^{\circ} \mathrm{C}$ & 612 \\
\hline dnaJ & 1110 & $\begin{array}{l}\text { dnaJ-259 } \\
\text { dnaJ-846 }\end{array}$ & $\begin{array}{l}\text { TTTGAAGATTTAGGCTCGTT } \\
\text { TAAAGAYGGCACTTTAATCG }\end{array}$ & $54^{\circ} \mathrm{C}$ & 588 \\
\hline luxS & 468 & $\begin{array}{l}\operatorname{luxS}-38 \\
\operatorname{luxS}-466\end{array}$ & $\begin{array}{l}\text { TGGATCACACYAAAGTCAAAG } \\
\text { AAACCCCCACTTCAGACCA }\end{array}$ & $54^{\circ} \mathrm{C}$ & 429 \\
\hline
\end{tabular}

Ta, annealing temperature.

aData from the complete genome of Helicobacter pylori 26695 (NC_000915). 
Table 3 Genetic distance values of individual and concatenated genes

\begin{tabular}{lllll}
\hline & \multicolumn{4}{l}{ Distances values } \\
\cline { 3 - 5 } Gene & $\begin{array}{l}\text { Sequence } \\
\text { (nt) }\end{array}$ & Range & Minimum & Mean \pm SE \\
\hline amiA & 576 & $0-0.052$ & 0.019 & $0.031 \pm 0.004$ \\
cgt & 558 & $0-0.044$ & 0.009 & $0.025 \pm 0.004$ \\
cpn60 & 555 & $0-0.039$ & 0.009 & $0.023 \pm 0.004$ \\
cpn70 & 588 & $0-0.040$ & 0.015 & $0.027 \pm 0.004$ \\
dnaJ & 564 & $0-0.061$ & 0.013 & $0.032 \pm 0.004$ \\
luxS & 405 & $0-0.078$ & 0.028 & $0.046 \pm 0.006$ \\
Concatenated & 3246 & $0-0.036$ & 0.024 & $0.030 \pm 0.002$ \\
\hline
\end{tabular}

a Minimum distances values between sequences from clones isolated from biopsies of different patients.

All housekeeping genes (amiA, cgt, cpn60, cpn70, dnaJ, and $\operatorname{lu} x S$ ) were specifically amplified for the bacterial species H. pylori with the primers designed in this study, none gene was amplified for the other bacterial species analyzed. Amplicons and partial gene sequences were 429-635 nt and 405-588 nt long, respectively (Table 2, Table 3).

Gene sequences from the $52 \mathrm{H}$. pylori clones and from H. pylori ATCC 49503 and ATCC 51932 were compared. All gene sequences from clones isolated from biopsies of different patients were different, whose gene distances ranged from 0.9 to $7.8 \%$ and the highest distance values were obtained from luxS. Distance rates calculated from concatenated sequences ranged from 2.4 to $3.6 \%$, with a mean distance of $3 \%$ (Table 3 ).

All clones isolated from the same patient showed identical sequences except in the case of B508S/B508T and B657A/B657C. Two different amiA and cpn60 sequences were detected from B508T clones: B508T2A, B508T-2B, B508T-3, B508T-5, and B508T-6 showed identical sequences, which were different to those from clone B508T-4. A unique nucleotide difference $(0.2 \%$ divergence) was detected in the gene amiA, meanwhile six nucleotide differences ( $1.1 \%$ divergence) were found in cpn60. B508S clones presented identical amiA and cpn60 sequences to those from B508T-4. However, on the basis of cgt, cpn70, dnaJ, and luxs sequence comparison, all B508T and B508S clones analyzed were identical. Phylogenetics trees for amiA, cpn60, luxS, cgt, cpn70, and dnaJ are shown in Figure 1A, B, C and Fig. S1A, B, C, respectively.

On the other hand, all six genes allowed to differentiate two groups of clones from B657A and B657C biopsies. A group was formed by B657A-1, B657A-2, and B657A-3 and another group by B657A-4 and all four clones from B657C (Fig. 1B, C and Fig. S1A, B, C), with the following intergroup distance rates: 3.6, $2.2,3.5,3.1,1.6$, and $7.3 \%$ for the genes amiA, cgt, cpn60, cpn70, dnaJ, and luxs, respectively. Moreover, two nucleotide differences $(0.3 \%$ divergence) were observed between B657A-4 and B657C clones for amiA (Fig. 1A).

The phylogenetic tree obtained from concatenated sequences $(3246 \mathrm{nt})$ is shown in Figure 2.

\section{Discussion}

In this study, specific PCR detection of H. pylori has been achieved for the housekeeping genes amiA, cgt, cpn60, cpn70, dnaJ, and luxS. These genes are potential candidates to detect $H$. pylori infection in gastric biopsies and other specimens (as gastric juice, stool, saliva, dental plaque, water, and food samples), together with others, mainly used for gastric biopsies, as ure $A$, vacA, 16S rRNA, and 23S rRNA genes, previously described $[17,18]$.

Sequencing of any of these six genes has allowed a clear differentiation between all clones isolated from different patients (0.9-7.8\% divergence). These results suggest that these housekeeping genes could be useful to elucidate the mode of transmission of $H$. pylori, an issue that is still controversial. While oral-oral, gastro-oral, and fecal-oral transmission are the most probable ways, H. pylori isolation from stools or the oral cavity is difficult and different environmental sources of $H$. pylori (food, animals, water) could play a role as reservoirs [10].

Two different strains isolated from the antral biopsy B657A were clearly detected by all genes with high distance values (1.6-7.3\%). One strain was represented by the identical clones B657A-1, -2 and -3 , and the other strain by the clone B657A-4, which was identical to clones isolated from B657C (corpus biopsy from the same patient), except in the case of amiA as mentioned below. This high divergence observed between both strains indicates an event of multiple infection in the antrum of the stomach. Horizontal gene transfer and genetic recombination by mixed infection is crucial for the acquisition of the high genetic diversity of $\mathrm{H}$. pylori [19]. Although some authors have detected mixed infections, different studies have reported very variable prevalence rates and little is known about the role of multiple infections on disease outcome $[8,20,21]$.

Figure 1 Consensus neighbor-joining phylogenetic trees obtained from 18 Helicobacter pylori sequences of genes amiA (A) cpn60 (B) and luxS (C). Bar, distance values as calculated by MEGA 6.0. Bootstrap values (>50\%) after 1000 replicates are shown as percentages. 

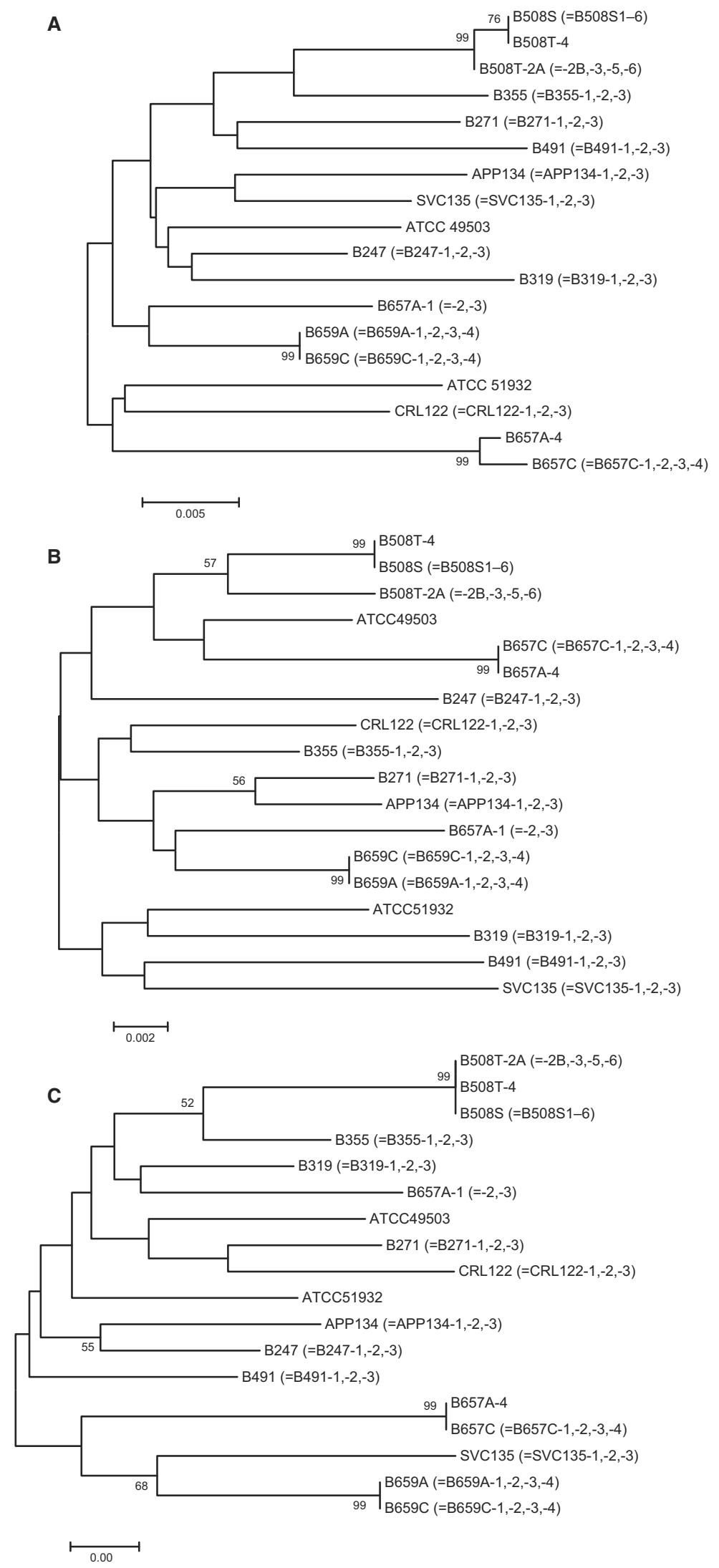


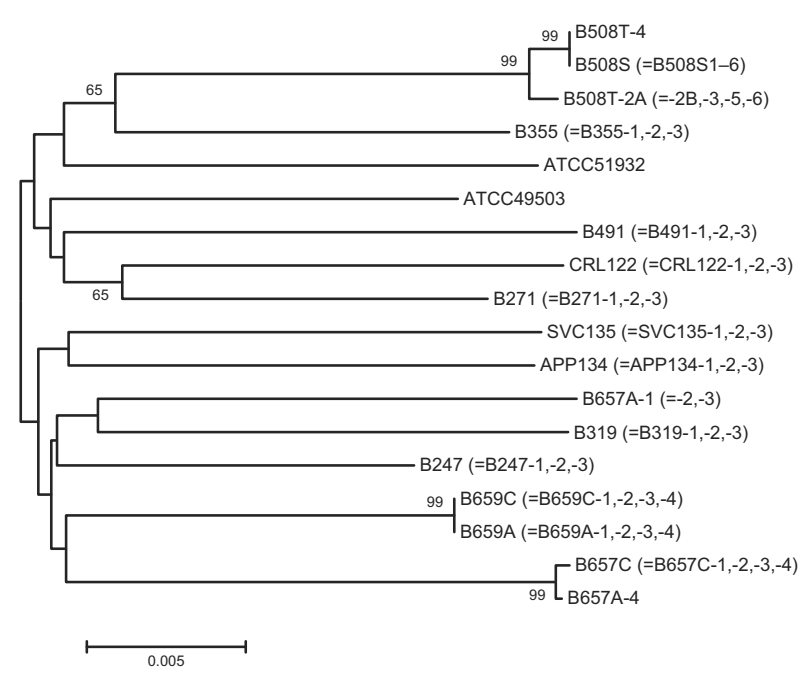

Figure 2 Consensus neighbor-joining phylogenetic tree obtained from amiA, cgt, cpn60, cpn70, dnaJ, and luxS concatenated sequences. Bar, distance of 0.005 substitutions per nucleotide position as calculated by MEGA 6.0. Bootstrap values (>50\%) after 1000 replicates are shown as percentages.

Although luxS gene showed the highest distance values, the genes amiA and cpn60 were more useful to discriminate clones isolated from the same biopsy and patient. Two different clones isolated from the sample B508T, gastric biopsy collected from tissue with adenocarcinoma, were only detected by partial sequencing of amiA and cpn60 (one and six nucleotide differences, respectively). One of both clones (B508T-4) was identical to clones isolated from B508S, gastric biopsy from the same patient but collected from normal tissue. On the other hand, two amiA nucleotide differences were observed between B657A-4 and B657C clones. These small differences between clones suggest events of microevolution rather than related to multiple infection. Evidences that $H$. pylori can establish a chronic infection after infection with a single strain have been described [22,23] and, recently, Linz et al. [24] have revealed that the mutation rate during the acute infection phase is over 10 times faster than during chronic infection.

Concatenation of amiA, cgt, cpn60, cpn70, dnaJ, and luxS partial sequences allowed us to conduct a multilocus sequence analyses (MLSA) approach (Fig. 2), which is useful to elucidate intra- and interspecies phylogenetic relationships $[25,26]$ and, in the case of $H$. pylori, the phylogeographic differentiation of bacterial populations associated to the migration of human populations $[6,27]$. Most of these genes have never been used for these purposes except dnaJ and, especially, cpn60, that is useful for microbial phylogeny, detection and identi- fication, ecology, and evolution through the analysis of the 555 bp region known as universal target (UT) analyzed in this study $[28,29]$.

In conclusion, H. pylori specific detection has been developed, all $H$. pylori clones isolated from different patients have been discriminated, and microevolution and mixed infection have been detected by means of amplification and sequencing of housekeeping genes amiA, cgt, cpn60, cpn70, dnaJ, and luxs for the first time. Further studies based on these housekeeping genes are needed to explore their potential applications for $H$. $p y$ lori detection in different specimens, the mode of transmission of H. pylori, the role associated to virulence or the phylogeographic differentiation of $\mathrm{H}$. pylori populations.

\section{Acknowledgements and Disclosures}

We would like to thank A. Flaqué for her contribution to this study. This work has been supported by a project from the Fundació La Marató de TV3 (20130831), Catalonia.

Competing interests: The authors have declared that no competing interests exist.

\section{Note}

The GenBank/EMBL/DDBJ accession numbers for the 11 amiA, cgt, cpn60, cpn70, dnaJ and luxs gene sequences are KU053341-58, KU053359-76, KU053377-94, KU053395-412, KU053413-30 and KU053431-48, respectively.

\section{References}

1 Correa P, Houghton J. Carcinogenesis of Helicobacter pylori. Gastroenterology 2007;133:659-72.

2 Mobley HLT, Mendz GL, Hazell SL. Helicobacter pylori: Physiology and Genetics. Washington (DC): ASM Press, 2001.

3 Mascellino MT, Margani M, Oliva A. Helicobacter pylori: determinant and markers of virulence. Dis Markers 2009;27:137-56.

4 Backert S, Clyne M. Pathogenesis of Helicobacter pylori infection. Helicobacter 2011;16(Suppl. 1):19-25.

5 González CA, Figueiredo C, Lic CB, Ferreira RM, Pardo ML, Ruiz Liso JM, et al. Helicobacter pylori cagA and vacA genotypes as predictors of progression of gastric preneoplastic lesions: a long-term follow-up in a high-risk area in Spain. Am J Gastroenterol 2011;106:867-74.

6 Linz B, Balloux F, Moodley Y, Manica A, Liu H, Roumagnac P, et al. An African origin for the intimate association between humans and Helicobacter pylori. Nature 2007;445:915-8.

7 Moodley Y, Linz B, Yamaoka Y, Windsor HM, Breurec S, Wu JY, et al. The peopling of the Pacific from a bacterial perspective. Science 2009;323:527-30.

8 Sheu SM, Sheu BS, Lu CC, Yang HB, Wu JJ. Mixed infections of Helicobacter pylori: tissue tropism and histological significance. Clin Microbiol Infect 2009;15:253-9. 
9 Talarico S, Gold BD, Fero J, Thompson DT, Guarner J, Czinn S, et al. Pediatric Helicobacter pylori isolates display distinct gene coding capacities and virulence gene marker profiles. J Clin Microbiol 2009;47:1680-8.

10 Khalifa MM, Sharaf RR, Aziz RK. Helicobacter pylori: a poor man's gut pathogen? Gut Pathog 2010;2:2.

11 Chaput C, Ecobichon C, Cayet N, Girardin SE, Werts C, Guadagnini $S$, et al. Role of AmiA in the morphological transition of H. pylori and in immune escape. PLoS Pathog 2006;2:e97.

12 Di Felice V, David S, Cappello F, Farina F, Zummo G. Is chlamydial heat shock protein 60 a risk factor for oncogenesis? Cell Mol Life Sci 2005;62:4-9.

13 McGee DJ, George AE, Trainor EA, Horton KE, Hildebrandt E, Testerman TL. Cholesterol enhances H. pylori resistance to antibiotics and LL-37. Antimicrob Agents Chemother 2011;55:2897-904.

14 Lario S, Ramírez-Lázaro MJ, Aransay AM, Lozano JJ, Montserrat $A$, Casalots $\dot{A}$, et al. microRNA profiling in duodenal ulcer disease caused by Helicobacter pylori infection in a Western population. Clin Microbiol Infect 2012;18:E273-82.

15 Tamura K, Stecher G, Peterson D, Filipski A, Kumar S. MEGA6: Molecular evolutionary genetics analysis version 6.0. Mol Biol Evol 2013;30:2725-9.

16 Villesen P. FaBox: an online toolbox for FASTA sequences. Mol Ecol Notes 2007;7:965-8.

17 Ramírez-Lázaro MJ, Lario S, Casalots A, Sanfeliu E, Boix L, García-Iglesias P, et al. Real-time PCR improves Helicobacter pylori detection in patients with peptic ulcer bleeding. PLoS One 2011;6:e20009.

18 Wang YK, Kuo FC, Liu CJ, Wu MC, Shih HY, Wang SS et al. Diagnosis of Helicobacter pylori infection: current options and developments. World J Gastroenterol 2015;21:11221-35.

19 Falush D, Kraft C, Taylor NS, Correa P, Fox JG, Achtman M, et al. Recombination and mutation during long-term gastric colonization by Helicobacter pylori: estimates of clock rates, recombination size, and minimal age. Proc Natl Acad Sci USA 2001;98:15056-61.

20 Raymond J, Thiberg JM, Chevalier C, Kalach N, Bergeret M, Labigne A, et al. Genetic and transmission analysis of Helicobacter pylori strains within a family. Emerg Infect Dis 2004;10:181621.

21 Ghose C, Pérez-Pérez GI, van Doorn LJ, Domínguez-Bello MG, Blaser MJ. High frequency of gastric colonization with multiple Helicobacter pylori strains in Venezuelan subjects. J Clin Microbiol 2005;43:2635-41.
22 Kennemann L, Didelot X, Aebischer T, Kuhn S, Drescher B Droege $\mathrm{M}$, et al. Helicobacter pylori genome evolution during human infection. Proc Natl Acad Sci USA 2011;108:5033-8.

23 Toita N, Yokota S, Fujii N, Konno M. Clonality analysis of Helicobacter pylori in patients isolated from several biopsy specimens and gastric juice in a Japanese urban population by random amplified polymorphic DNA fingerprinting. Gastroenterol Res Pract 2013;2013:721306.

24 Linz B, Windsor HM, McGraw JJ, Hansen LM, Gajewski JP, Tomsho LP, et al. A mutation burst during the acute phase of Helicobacter pylori infection in humans and rhesus macaques. Nat Commun 2014;5:4165.

25 Gevers D, Cohan FM, Lawrence JG, Spratt BG, Coenye T, Feil EJ, et al. Opinion: re-evaluating prokaryotic species. Nat Rev Microbiol 2005;3:733-9.

26 Tindall BJ, Rosselló-Móra R, Busse HJ, Ludwig W, Kämpfer P. Notes on the characterization of prokaryote strains for taxonomic purposes. Int J Syst Evol Microbiol 2010;60:249-66.

27 Suzuki R, Shiota S, Yamaoka Y. Molecular epidemiology, population genetics, and pathogenic role of Helicobacter pylori. Infect Genet Evol 2012;12:203-13.

28 Hill JE, Paccagnella A, Law K, Melito PL, Woodward DL, Price $\mathrm{L}$, et al. Identification of Campylobacter spp. and discrimination from Helicobacter and Arcobacter spp. by direct sequencing of PCR-amplified cpn60 sequences and comparison to cpnDB, a chaperonin reference sequence database. J Med Microbiol 2006;55:393-9.

29 Miñana-Galbis D, Farfán M, Lorén JG, Fusté MC. The reference strain Aeromonas hydrophila CIP 57.50 should be reclassified as Aeromonas salmonicida CIP 57.50. Int J Syst Evol Microbiol 2010;60:715-7.

\section{Supporting Information}

Additional Supporting Information may be found in the online version of this article:

Figure S1 Consensus neighbor-joining phylogenetic trees obtained from $18 \mathrm{H}$. pylori sequences of genes $\mathrm{cgt}$ (A), cpn70 (B), and dnaJ (C). Bar distance values as calculated by MEGA 6.0. Bootstrap values $(>50 \%)$ after 1000 replicates are shown as percentages.

Table S1 Helicobacter pylori complete genome sequences used to primer design. 
Figure S1 (A)

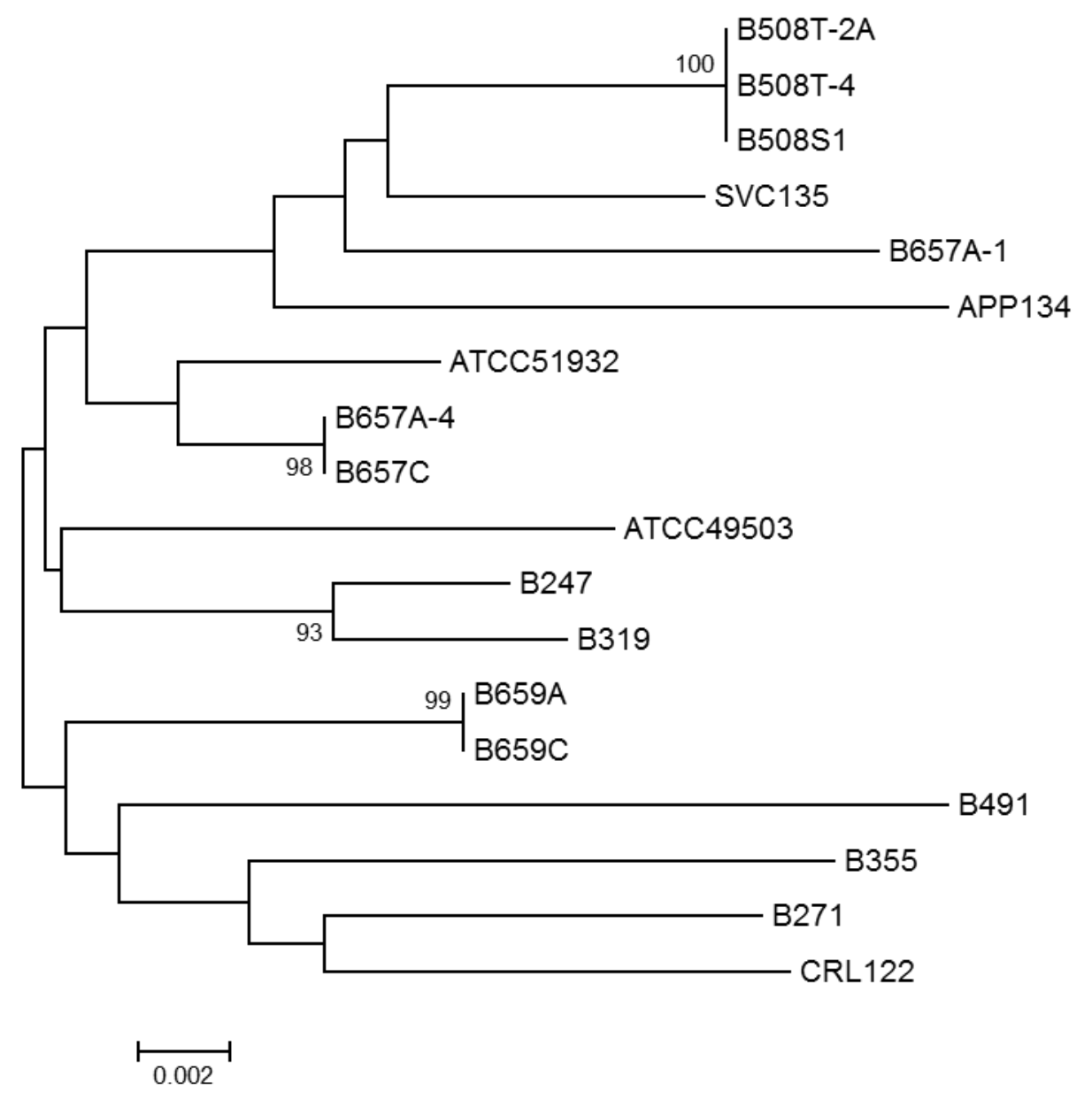


Figure S1 (B)

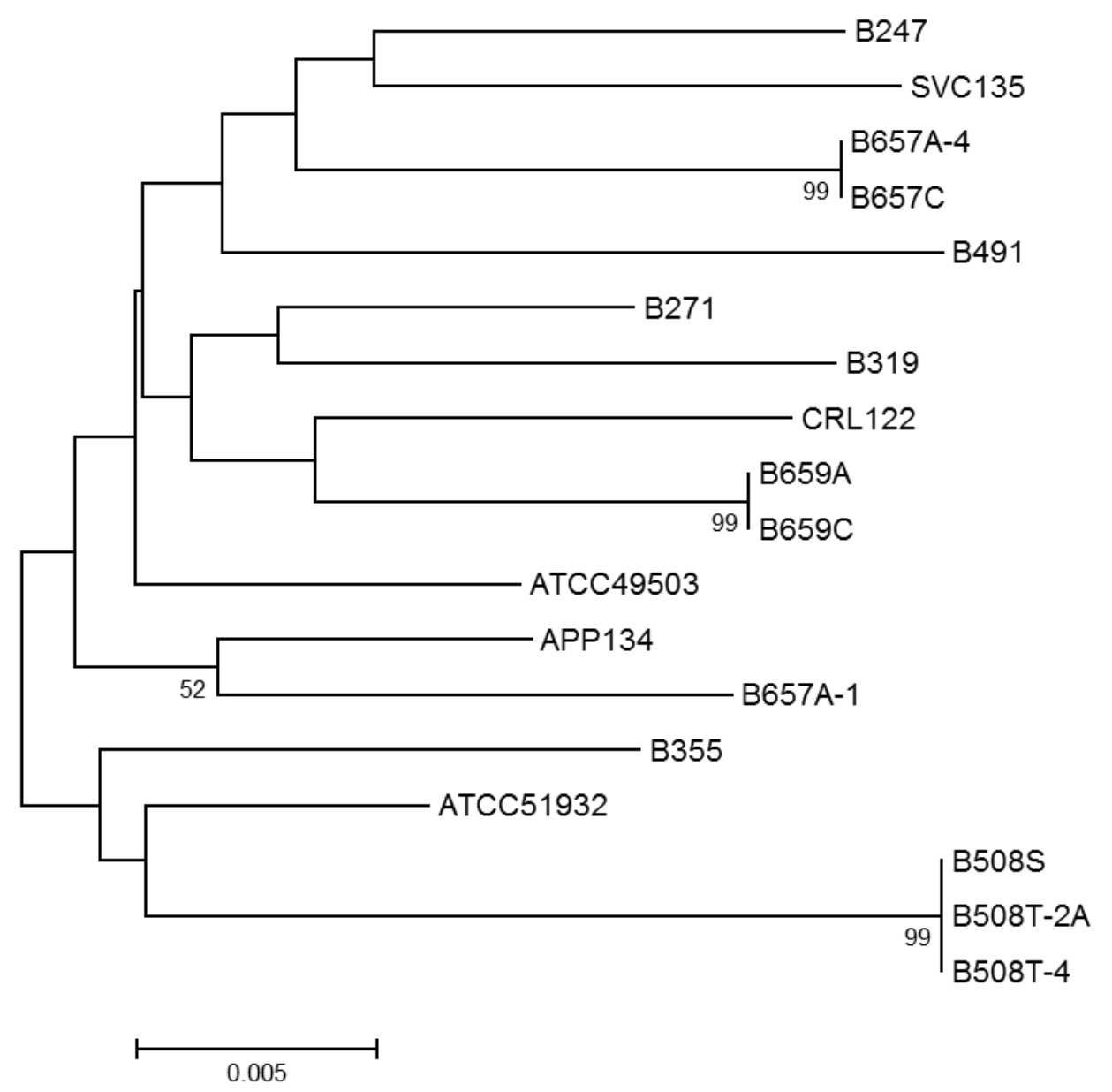


Figure S1 (C)

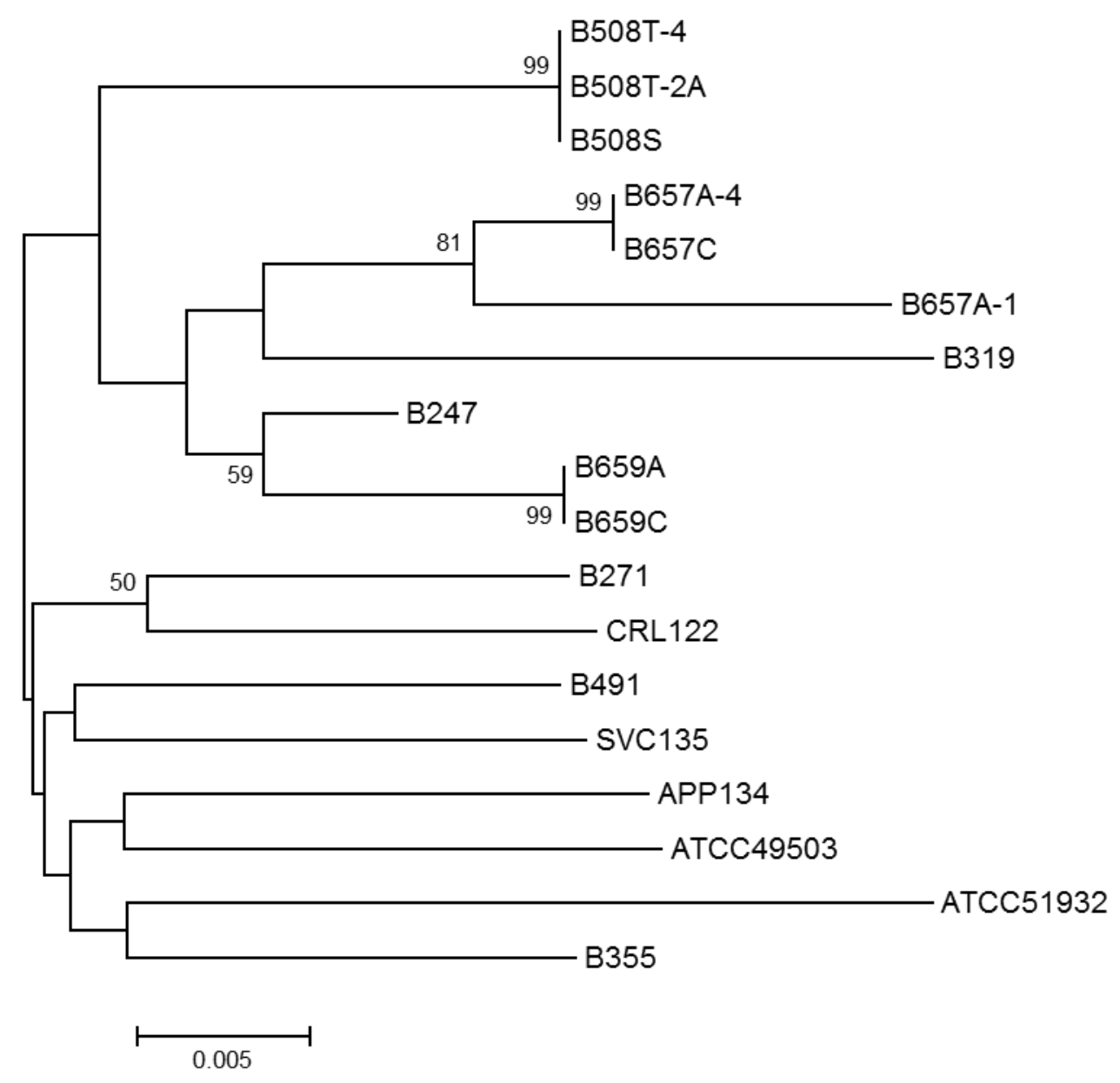


Table S1 H. pylori complete genome sequences used to primer design

\section{$H$. pylori strain Reference sequence number}

51

CP000012

52

CP001680

$83 \quad$ CP002605

$908 \quad$ CP002184

$2017 \quad$ CP002571

$2018 \quad$ CP002572

$26695 \quad$ CP003904

$26695 \quad$ NC_000915

35A CP002096

Aklavik117 CP003483

Aklavik86 CP003476

B38 NC_012973

B8 NC_014256

Cuz20 CP002076

ELS37 CP002953

F16 AP011940

F30 AP011941

F32 AP011943

F57 AP011945

G27 NC_011333

Gambia94/24 CP002332

HPAG1 NC_008086

HUP-B14 NC_017733

India7 CP002331

J99 NC_000921

Lithuania75 CP002334

P12 NC_011498

PeCan18 NC_017742

PeCan4 NC_014555

Puno120 CP002980

Puno135 CP002982

Rif1 CP003905

Rif2 CP003906

Sat464 CP002071

Shi112 NC_017741

Shi169 NC_017740

Shi417 NC_017739

Shi470 NC_010698

SJM180 NC_014560

SNT49 CP002983

SouthAfrica7 CP002336

v225d CP001582

XZ274 CP003419 